\title{
Redshift-Space Distortions and $f(z)$ from Group-Galaxy Correlations
}

\author{
F. G. Mohammad ${ }^{1}$, S. de la Torre ${ }^{2}$, L. Guzzo ${ }^{1}$, D. Bianchi ${ }^{1}$ and \\ J. A. Peacock ${ }^{3}$ \\ ${ }^{1}$ INAF-Osservatorio Astronomico di Brera, IT-23807, Merate (LC), Italy \\ email: faizan.mohammad@brera.inaf.it \\ ${ }^{2}$ LAM - Laboratoire dAstrophysique de Marseille, 13388 Marseille France \\ ${ }^{3}$ Institute for Astronomy-The University of Edinburgh, Edinburgh EH9 3HJ, U.K.
}

\begin{abstract}
We investigate the accuracy achievable on measurements of the the growth rate of structure $f(z)$ using redshift-space distortions (RSD), when (a) these are measured on the group-galaxy cross correlation function; (b) the latter is expanded over a modified version of the conventional spherical armonics, "truncated multipole moments". Simulation results give first indications that this combination can push systematic errors on $f(z)$ below $3 \%$, using scales $r \geqslant 10 h^{-1} \mathrm{Mpc}$.
\end{abstract}

Keywords. Large-scale structure, cosmological parameters, clustering

Linear "Redshift-Space Distortions" (RSD) in two-point galaxy correlations (2PCF) measure the growth rate of structure $f(z)$ and represent a powerful test of gravity on cosmological scales. Yet, modelling the observed anisotropy of clustering to extract the linear RSD signal is complicated by non-linear contributions: the perused Dispersion Model applied to the galaxy $2 \mathrm{PCF}$ introduces systematic effects $\sim 10 \%$ on the recovered values of $f$ [e.g. Bianchi et al. (2012)]. Together with building more realistic models, improvement could be obtained by (a) choosing specific tracers that are less sensitive to non-linear contributions; (b) using a different two-point statistics. We show here results using the group-galaxy cross correlation ( $\mathrm{CCF})$, together with a modified multipole expansion, the "truncated multipole moments", $\hat{\xi}^{(l)}$, which provide the practical advantage of allowing the exclusion of small scales dominated by non-linear distortions.

We first adapt the linear model [Kaiser (1987), Hamilton (1992)] to the group-galaxy $\mathrm{CCF}$

$$
\xi_{c r, L i n}^{s}\left(r_{p}, \pi\right)=\sum_{l=0,2,4} \xi_{c r, L i n}^{s,(l)}(s) L_{l}(\mu)
$$

with $\xi_{c r, L i n}^{s,(l)}(s)$ being the multipoles of the 2PCF. This expression depends directly on the parameter $\beta_{\text {gal }}(z)=f(z) / b_{\text {gal }}(z)$, the relative bias $b_{12}=b_{\text {gal }} / b_{\text {gr }}$ and the real-space $2 \mathrm{PCF} \xi(r)$. We define the truncated multipoles of the $2 \mathrm{PCF}$ as

$$
\hat{\xi}^{s,(l)}(s)=\frac{2 l+1}{2} \int_{-\bar{\mu}}^{+\bar{\mu}} \xi^{s}(s, \mu) L_{l}(\mu) d \mu,
$$

where $\bar{\mu}=\sqrt{1-\left(\overline{r_{p}} / s\right)^{2}}$, and $\bar{r}_{p}$ is the minimum transverse scale to be included in the fit. Standard multipoles $\xi^{s,(l)}$ would correspond to $\bar{\mu}=1$. We fit to either the full $2 \mathrm{PCF}$ or the multipoles the standard dispersion model, in which the linear model above is empirically corrected for non-linearities through a scale-independent pairwise velocity dispersion [Peacock \& Dodds (1994)]. 


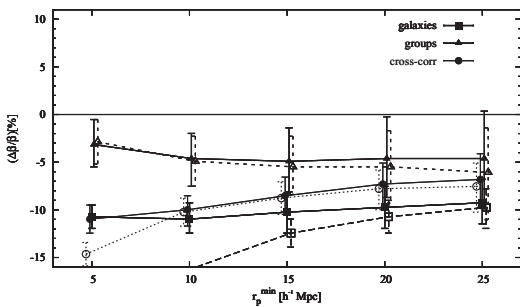

(a) Anisotropic $2 \mathrm{PCF} \xi^{s}\left(r_{p}, \pi\right)$

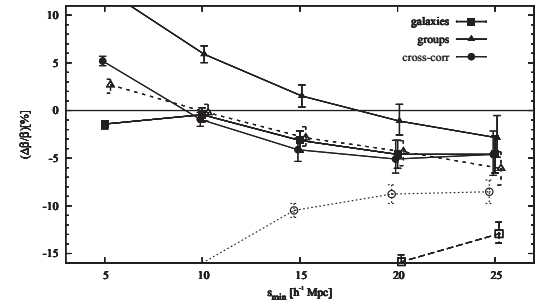

(b) Standard multipoles $\xi^{s,(l)}(s)$

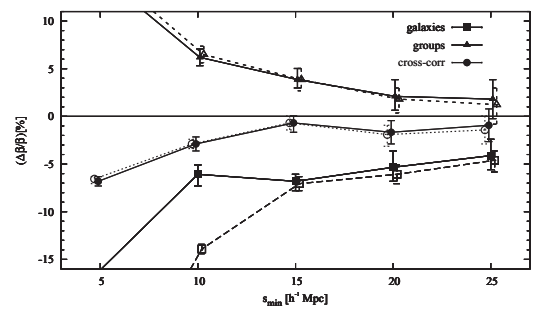

(c) Truncated multipoles $\hat{\xi}^{s,(l)}(s)$

Figure 1. Results of fitting the $2 \mathrm{PCF}$ and $\mathrm{CCF}$ of galaxies and groups, using the techniques discussed in the text.

All this is tested over an ensamble of simulated galaxy/halo catalogues obtained from the MultiDark Run1 (MDR1) n-body simulation [Prada et al. (2012)], from which we compute both ensemble statistical errors and systematic differences with respect to the known simulation growth rate (measured through $\beta$ ).

Figure 1 compares systematic and statistical errors obtained on the parameter $\beta$ when using the $2 \mathrm{PCF}$ for galaxies (squares), groups (i.e. halos, triangles) and their CCF (circles) and fitting respectively the full two-point functions and the standard or truncated multipoles (panels a, b, c). Continuous lines use the full dispersion model while dashed lines correspond to fits using the pure linear model. The top-left panel simply confirms the results of Bianchi et al. (2012) for the auto-correlation function, i.e. a general underestimation of the growth rate, which becomes smaller for groups, i.e. higher-mass halos. Fitting the standard multipoles $\xi^{s,(l)}$, instead, yields systematic errors that are very sensitive to the range of scales included in the fit for all methods, reducing to within $\pm 5 \%$ for scales $\gtrsim 15 h^{-1} \mathrm{Mpc}$. We interpret this as the effect of the projection of the $2 \mathrm{PCF}$ on Legendre polynomials which spreads over all scales $s$, non linearities originally confined to small transverse scales $r_{p}$. We defined the truncated multipoles $\hat{\xi}^{s,(l)}$ specifically to avoid this. The third panel in fact shows an even different trend, with the cross correlation providing a stable systematic error on $\beta$, confined within a few percent when using scales $\gtrsim 10 h^{-1} \mathrm{Mpc}$. In all cases the discrepancy between the dispersion model and the linear one decreases moving to larger scales. In Mohammad et al. (2014, in preparation) we also discuss the role of properly accounting for the covariance matrix.

\section{References}

Bianchi, D., Guzzo, L., Branchini, E., et al. 2012, MNRAS, 427, 2420

Hamilton, A. J. S. 1992, APJL, 385, L5

Kaiser, N. 1987, MNRAS, 227, 1

Peacock, J. A. \& Dodds, S. J. 1994, MNRAS, 267, 1020

Prada, F., et al., MNRAS, 423, 3018 